\title{
Intermediate W Bosons, Gauge Fields, and the Nonleptonic Weak Decays*
}

\author{
YUкIO TOMOZAWA \\ Randall Laboratory of Physics, \\ University of Michigan, Ann Arbor, Michigan 48104
}

Received July 27,1970

Following the analysis of Nishijima and Sato, we use the chiral symmetric gauge field model to compute the $s$-wave amplitudes of the nonleptonic weak decays. It is pointed out that the weak interaction Lagrangian which has been derived from the assumption of octet $\mathrm{W}$ bosons is consistent with experiment. The $p$-wave amplitudes of the nonleptonic hyperon decay are obtained by using the sum rule that has been derived by the author. The result for both $s$ - and $p$-wave amplitudes, which is a theoretical prediction with no adjustable parameter, is in fair accord with the experimental data.

\section{INTRODUCTION}

Recently, we proposed a model [1] of the weak interaction Lagrangian based on intermediate vector bosons (W bosons) that transform as an $\mathrm{SU}_{3}$ octet or nonet. By imposing a phenomenological requirement that the $\Delta S=2$ transitions be absent in the lowest order of the Fermi coupling constant $G_{\mathrm{F}}$, we were led, in a natural way, to a theory which is intrinsically $C P$ violating. The effective Lagrangian thus obtained has no 27 component, and the term responsible for nonleptonic weak decays is given by ${ }^{1}$

$$
\begin{aligned}
-L_{\mathrm{W}}^{|\Delta S|=1} & =\xi \frac{G_{\mathrm{F}}}{\sqrt{2}}\left(J_{\mu} J_{\mu}\right)_{3}^{2} \sin \theta \cos \theta+\text { h.c. } \\
& =\xi \sqrt{2} G_{\mathrm{F}} d_{6 \alpha \beta} J_{\mu}{ }^{\alpha} J_{\mu}{ }^{\beta} \sin \theta \cos \theta
\end{aligned}
$$

* Work supported in part by the United States Atomic Energy Commission.

${ }^{1}$ The corresponding terms in Ref. [1] have phase factors which reflect the $C P$ violating nature of the basic Lagrangian. As was discussed in Ref. [1], however, these phases are unobservable as far as a process of order $G_{F}$ is concerned: we may remove them either by choosing the arbitrary phase in a suitable way or by redefining the phases of the basis states of the $S U_{3}$ representation. 
where

$$
\left(J_{\mu}\right)_{b}^{a}=\sum_{\substack{\alpha=1 \\ \text { (or } 01}}^{8}\left(\lambda_{\alpha}\right)_{b}^{a} J_{\mu}^{\alpha}, \quad(a, b=1,2,3)
$$

are the octet or nonet hadronic $V-A$ currents

$$
J_{\mu}=V_{\mu}+A_{\mu}
$$

$\lambda_{\alpha}, d_{\alpha \beta \gamma}$, and $\theta$ are the Gell-Mann spin matrix, the symmetric coefficients of the $S U_{3}$ group, and the Gell-Mann-Levy-Cabbibo (GLC) angle [2], respectively; and

$$
\begin{aligned}
\xi & =\sqrt{3} \text { for the model with octet } \mathrm{W} \text { bosons, } \\
& =\sqrt{2} \text { for the model with nonet } \mathrm{W} \text { bosons. }
\end{aligned}
$$

Notice that the Cabibbo Lagrangian gives $\xi=1$ if the octet is constructed by adding a component of neutral currents or $\xi==3 / 5$ if the octet part is extracted out of the charged current-current interaction.

A long-standing problem of nonleptonic weak decay in the current-current Lagrangian is whether the suppression by the GLC angle [3]

$$
\sin \theta \cos \theta=0.23
$$

can be compensated by a dynamical enhancement. The answer to this question was once negative [4], but recently the possibility of an affirmative answer was suggested by Nishijima and Sato [5]. Assuming the chiral symmetric gauge field model and field-current identity, they assert that the mixing effect of the gauge fields gives a desired enhancement. Since, however, it seems that some terms were neglected in their analysis, we reinvestigate the gauge field model, hoping to be able to determine which of the weak interaction models mentioned above is consistent with the experimental data for the $s$-wave amplitudes (Section 2). Section 3 deals with the $\mathrm{K}_{1}{ }^{0} \rightarrow 2 \pi$ decay and Section 4 is devoted to a discussion of the $p$-wave amplitudes.

\section{The s-WAVE Hyperon Decay}

As was pointed out in Ref. [5], we have to consider the mixing of the vector field,

$$
v_{\mu}{ }_{\mu}^{\mathbf{K}} \equiv\left(v_{\mu}\right)_{3}^{1} \equiv=\frac{1}{\sqrt{2}} v_{\mu \nu}^{4-i 5},
$$


and the axial vector field,

$$
a_{i \mu}{ }^{\pi}=\left(a_{\mu}\right)_{2}^{1} \equiv \frac{1}{\sqrt{2}} a_{\mu}^{1-i 2},
$$

as far as the parity-violating ( $s$-wave) amplitudes of the nonleptonic weak decays are concerned. Following the prescription of the chiral symmetric gauge field model [5-8], we may write down the relevant part of the Lagrangian in the following form,

$$
L=L_{\mathrm{G}}+L_{\mathrm{M}}+L_{\mathrm{W}}
$$

where

$$
\begin{aligned}
-L_{\mathbf{G}}= & \frac{1}{2}\left\{\left(v_{\mu \nu}^{\mathrm{K}}\right)^{\dagger} \iota_{\mu \nu}^{\mathrm{K}}+\left(a_{\mu \nu}^{\pi}\right)^{\dagger} a_{\mu \nu}^{\pi}\right\}+m_{v}{ }^{2}\left(v_{\mu}{ }^{\mathrm{K}}\right)^{\dagger} v_{\mu}{ }^{\mathrm{K}}+m_{a}{ }^{2}\left(a_{\mu}{ }^{\pi}\right)^{\dagger} a_{\mu}{ }^{\pi} \\
& +\left(\partial_{\mu} \varphi-\frac{g}{\sqrt{2} f_{\mathbf{0}}} a_{\mu}{ }^{\pi}\right)^{\dagger}\left(\partial_{\mu} \varphi-\frac{g}{\sqrt{2} f_{0}} a_{\mu}{ }^{\pi}\right), \\
-L_{\mathbf{M}}= & -\frac{g}{\sqrt{2}} \mathscr{J}_{\mu}^{\mathbf{4}}{ }^{+i 5} v_{\mu}{ }^{\mathbf{K}}-\frac{g}{\sqrt{2}} \mathscr{J}_{5 \mu}^{1+i 2} a_{\mu}{ }^{\pi}+\text { h.c. },
\end{aligned}
$$

and

$$
-L_{\mathrm{W}}=h\left(v_{\mu}{ }^{\mathrm{K}}\right)^{+\frac{1}{+}} a_{\mu}{ }^{\pi}+\text { h.c. }
$$

In the gauge field Lagrangian $L_{\mathrm{G}}$, we use the notation

$$
\begin{aligned}
& v_{\mu \nu}^{\mathrm{K}}=\partial_{\mu} v_{\nu}{ }^{\mathrm{K}}-\partial_{\nu} v_{\mu \nu}{ }^{\mathrm{K}}-\frac{i g}{\sqrt{2}}\left\{\left[v_{\mu}, v_{\nu}\right]^{\mathrm{K}}+\left[a_{\mu}, a_{\nu}\right]^{\mathrm{K}}\right\}, \\
& a_{\mu \nu}^{\pi}=\partial_{\mu} a_{\nu}{ }^{\pi}-\partial_{\nu} a_{\mu}{ }^{\pi}-\frac{i g}{\sqrt{2}}\left\{\left[v_{\mu}, a_{\nu}\right]^{\pi}+\left[a_{\mu}, v_{\nu}\right]^{\pi}\right\}
\end{aligned}
$$

the mass terms obey $S U(2) \times S U(2)$ symmetry, i.e.,

$$
m_{a}=m_{\rho}
$$

and the third term breaks that symmetry, $\varphi$ being the unrenormalized pion field. The vector current $\mathscr{J}_{\mu}^{4+i 5}$ and the axial vector current $\mathscr{J}_{5 \mu}^{1+i 2}$ in the matter Lagrangian $L_{\mathrm{M}}$ consist of hadronic fields. In the weak interaction Lagrangian $L_{\mathrm{W}}$, the coupling strength $h$ is given by

$$
h=\xi \sqrt{2} G_{\mathrm{F}} \sin \theta \cos \theta \frac{m_{v}{ }^{2}}{g} \frac{m_{a}{ }^{2}}{g},
$$


where use is made of the relation

$$
2 d_{6 \alpha \beta} J_{\mu \alpha}{ }^{\alpha} J_{\mu}{ }^{\beta}=J_{\mu}^{4-i 5} J_{\mu}^{1+i 2}+\text { h.c. }-2 J_{\mu}{ }^{6}\left(J_{\mu}{ }^{3}+\frac{1}{\sqrt{3}} J_{\mu}{ }^{8}\right),
$$

and the field-current identity [9]

$$
V_{u}^{4+i 5}=\frac{\sqrt{2} m_{v}^{2}}{g} v_{k \mu} \mathrm{K}
$$

and

$$
A_{\mu}^{1+i 2}=\frac{\sqrt{2} m_{u}{ }^{2}{ }^{2}}{g} a_{\mu}{ }^{\pi} .
$$

Incidentally, we notice that if we use the chiral-covariant combination for the second term of Eq. (8),

$$
-\frac{g^{\prime}}{\sqrt{2}} \mathscr{J}_{5 \mu}^{1+i 2}\left(a_{u}{ }^{\pi}-\frac{\sqrt{2} f_{0}}{g} \partial_{\mu} \varphi\right),
$$

and at the same time for Eq. (14A),

$$
\mathrm{A}_{\mu}^{1+i 2}=\frac{\sqrt{2} m_{a^{2}}{ }^{2}}{g}\left(a_{\mu}{ }^{\pi}-\frac{\sqrt{2} f_{0}}{g} \partial_{\mu} \varphi\right),
$$

then our result will be unchanged, except for the reversal of the sign of the PCAC constant.

In computing the decay amplitude, we first diagonalize [5] the bilinear terms of the gauge fields in $L_{\mathrm{G}}+L_{\mathrm{W}}$ by the following transformation;

$$
\begin{aligned}
v_{\mu}{ }^{\mathrm{K}} & =\mathrm{K}_{\mu}{ }^{*}+\lambda \mathrm{A}_{1 \mu}+c_{1} \partial_{\mu} \pi, \\
a_{\mu}{ }^{\pi} & =-\lambda \mathrm{K}_{\mu}{ }^{*}+\mathrm{A}_{1 \mu}+c_{2} \partial_{\mu} \pi, \\
\varphi & =Z^{1 / 2} \pi,
\end{aligned}
$$

where $\lambda, c_{1}$, and $c_{2}$ are constants to be determined by the diagonalization condition, and the renormalization constant $Z$ is necessary in order to have a correct coeffcient for the kinetic energy term of the physical pion field $\pi$ after the mixing, Eq. (15). The coefficients thus obtained are, in the lowest order of the weak interaction,

$$
\begin{gathered}
\lambda=\frac{h}{m_{\mathrm{A}_{1}}^{2}-m_{\mathrm{K} *}^{2}}, \\
c_{1}=-\frac{h}{m_{\mathrm{K}^{*}}^{2} m_{\mathrm{A}_{1}}}, \quad c_{2}=\frac{1}{m_{\mathrm{A}_{1}}},
\end{gathered}
$$


and

$$
Z=2 \text {, }
$$

where

$$
\begin{aligned}
& m_{\mathrm{K}^{*}}^{2}=m_{v}{ }^{2} \\
& m_{\mathrm{A}_{1}}^{2}=m_{a^{2}}{ }^{2}+\frac{g^{2}}{2 f_{0}{ }^{2}},
\end{aligned}
$$

and the Weinberg mass relation [10]

$$
m_{\mathrm{A}_{1}}^{2}=2 m_{a}^{2},
$$

i.e.,

$$
\frac{g^{2}}{2 f_{0}^{2}}=m_{a}^{2},
$$

has been used. The fields $K_{\mu}{ }^{*}$ and $A_{1 \mu}$ correspond to the physical particles $K^{*}$ and $A_{1}$ respectively, and should satisfy the conditions

$$
\left\langle 0\left|\mathbf{K}_{\mu}^{*}\right| \pi\right\rangle=0
$$

and

$$
\left\langle 0\left|\mathrm{~A}_{1 \mu}\right| \pi\right\rangle=0 \text {. }
$$

Using these, together with Eqs. (14)-(16) and (20), we have

$$
\begin{aligned}
\left\langle 0\left|A_{\mu}^{1-i 2}\right| \pi\right\rangle & =\frac{\sqrt{2} m_{a}{ }^{2}}{g}\left\langle 0\left|a_{\mu}{ }^{\pi}\right| \pi\right\rangle \\
& =\frac{m_{a}}{g}\left\langle 0\left|c_{l l} \pi\right| \pi\right\rangle,
\end{aligned}
$$

which is nothing but the KSRF relation [11],

$$
g f_{\pi}=m_{\rho}=m_{\alpha},
$$

where $f_{\pi}$ stands for the $\pi \rightarrow \mu \nu$ decay constant.

The transformation (15) leads to the effective Hamiltonian for parity-violating decay processes,

$$
\begin{aligned}
H_{\mathrm{int}}^{\mathrm{pv}}= & -\frac{g}{\sqrt{2}} \mathscr{J}_{\mu}^{4+i 5}\left(\mathrm{~K}_{\mu}{ }^{*}+\frac{h}{m_{\mathrm{A}_{\mathrm{L}}}^{2}-m_{\mathrm{K}^{*}}^{2}} A_{1_{\mu}}-\frac{h}{m_{\mathrm{K}^{*}}^{2} m_{\mathrm{A}_{1}}} \partial_{\mu} \pi\right) \\
& -\frac{g^{\prime}}{\sqrt{2}} \mathscr{J}_{5 \mu}^{1+i 2}\left(-\frac{h}{m_{\mathrm{A}_{1}}^{2}-m_{\mathrm{K}}^{2}} \mathrm{~K}_{\mu}{ }^{*}+\mathrm{A}_{1, \mu}+\frac{1}{m_{\mathrm{A}_{1}}} \partial_{\mu} \pi\right)+\text { h.c. }
\end{aligned}
$$


The $s$-wave amplitude of the nonleptonic hyperon decay $\mathrm{Y} \rightarrow \mathrm{B} \pi^{-}$then may be computed as

$$
\begin{aligned}
\left\langle\mathrm{B} \pi^{-}\left|H_{\mathrm{int}}^{\mathrm{pv}}\right| \mathrm{Y}\right\rangle= & \left\{\frac{h}{m_{\mathrm{K} *}^{2} m_{\Lambda_{1}}}\left\langle\mathrm{~B}\left|\frac{g}{\sqrt{2}} \mathscr{J}_{\mu}^{4+i 5}\right| \mathrm{Y}\right\rangle\right. \\
& \left.-\frac{1}{m_{\mathrm{A}_{1}}}\left\langle\mathrm{~B}\left|\frac{g^{\prime}}{\sqrt{2}} \mathscr{J}_{5, \mu}^{1+i 2}\right| \mathrm{Y}\right\rangle\right\}\left\langle\pi^{-}\left|\partial_{\mu} \pi\right| 0\right\rangle
\end{aligned}
$$

The matrix elements in Eq. (24) are estimated by using the equation of motion for the $v_{\mu}{ }^{\mathrm{K}}$ and $a_{\rho}{ }^{\pi}$ fields,

$$
-\hat{c}_{\nu} v_{v u}^{\mathrm{K}}+m_{\mathrm{K}}^{\mathrm{Q} * c_{\mu}{ }^{\mathrm{K}}}=\frac{g}{\sqrt{2}} \mathscr{J}_{\mu}^{\mathrm{A}}{ }^{-i 5}-h a_{\mu}{ }^{\pi},
$$

and

$$
-\hat{c}_{\nu} a_{\nu \mu}^{\pi}+m_{\mathrm{A}_{1}}^{2} a_{\mu}{ }^{\pi}--\frac{g}{\sqrt{2} f_{0}} Z^{1 / 2} \hat{C}_{\mu} \pi=\frac{g^{\prime}}{\sqrt{\overline{2}}} \mathscr{J}_{5 \mu}^{1-i 2}-h_{\mu_{\mu}}{ }^{\mathrm{K}}
$$

i.e.,

$$
\begin{aligned}
\left\langle\mathrm{B}\left|\frac{g}{\sqrt{2}} \mathscr{J}^{4+i 5}\right| \mathrm{Y}\right\rangle & =m_{\mathrm{K}^{*}}^{2}\left\langle\mathrm{~B}\left|\left(v_{\mu}{ }^{\mathrm{K}}\right)^{\dagger}\right| \mathrm{Y}\right\rangle \\
& =\frac{g}{\sqrt{2}}\left\langle\mathrm{~B}\left|V_{\mu}^{4+i 5}\right| \mathrm{Y}\right\rangle
\end{aligned}
$$

and

$$
\begin{aligned}
\left\langle\mathrm{B}\left|\frac{g^{\prime}}{\sqrt{2}} \mathscr{J}_{5 \mu}^{1+i 2}\right| \mathrm{Y}\right\rangle & =m_{\mathrm{A}_{1}}^{2}\left\langle\mathrm{~B}\left|\left(a_{\mu}{ }^{\pi}\right)^{\dagger}\right| \mathrm{Y} ;+h\left\langle\mathrm{~B}\left|\left(v_{\mu}{ }^{\mathrm{K}}\right)^{\dagger}\right| \mathrm{Y}\right\rangle\right. \\
& =\left(-\frac{m_{\mathrm{A}_{1}}^{2}}{m_{\mathrm{A}_{1}}^{2}-m_{\mathrm{K}^{*}}^{2}}+1\right) h\left\langle\mathrm{~B} ;\left(c_{\mu}{ }^{\mathrm{K}}\right)^{\dagger} \mid \mathrm{Y}\right\rangle \\
& =-\frac{g}{\sqrt{2}} \frac{h}{m_{\mathrm{A}_{1}}^{2}-m_{\mathrm{K}^{*}}^{2}}\left\langle\mathrm{~B}\left|V_{\mu}^{4+i 5}\right| \mathrm{Y}\right\rangle
\end{aligned}
$$

where Eq. (15) has been used in the derivation of Eq. (27). The matrix element of the kinetic energy terms $\partial_{v} v_{\nu \mu}^{\mathrm{K}}$, etc., were omitted because they vanish when multiplied by $\left\langle\pi^{-}\left|\partial_{\mu} \pi\right| 0_{r}\right.$,

$$
\begin{aligned}
\left\langle\mathrm{B}\left|\partial_{\nu} v_{\nu \mu}^{\mathrm{K}}\right| \mathrm{Y}\right\rangle\left\langle\pi^{-}\left|\partial_{\mu} \pi\right| 0\right\rangle & =-i p_{\mu}{ }^{\pi}\left\langle\mathrm{B}\left|\partial_{\nu} v_{v \mu}^{\mathrm{K}}\right| \mathrm{Y}\right\rangle\left\langle\pi^{-}|\pi| 0\right\rangle \\
& =-\left\langle\mathrm{B}\left|\partial_{\mu} \partial_{\nu} v_{\nu \mu}^{\mathrm{K}}\right| \mathrm{Y}\right\rangle\left\langle\pi^{-}|\pi| 0\right\rangle \\
& -0 \text { for } p_{\nu \mu}{ }^{\mathrm{Y}}-p_{\mu}{ }^{\mathbf{B}}+p_{\mu}{ }^{\pi}
\end{aligned}
$$


$p_{\mu}{ }^{\mathrm{X}}$ being the four momentum of particle $\mathrm{X}$. Incidentally, we point out that it follows from Eqs. (15) and (25) that

$$
\left\langle\pi^{-}\left|\mathscr{J}^{4-i 5}\right| 0\right\rangle=\left\langle\pi^{-}\left|\mathscr{Z}_{5 \mu}^{1-i 2}\right| 0\right\rangle=0,
$$

as far as the last terms of Eq. (10) are neglected in computing the matrix elements $\left\langle\pi^{-}\left|\partial_{\nu} v_{v \mu}^{4-i 5}\right| 0\right\rangle$, etc.

Coming back to the computation of the matrix element of the decay $\mathrm{Y} \rightarrow \mathrm{B} \pi^{-}$, we obtain

$$
\begin{aligned}
\left\langle\mathrm{B} \pi^{-}\right. & \left.\left|H_{\mathrm{int}}^{\mathrm{pv}}\right| \mathrm{Y}\right\rangle \\
& =i N A\left(\mathrm{Y}_{-}\right) \bar{u}_{\mathrm{B}}\left(p^{\mathrm{B}}\right) u_{\mathrm{Y}}\left(p^{\mathrm{Y}}\right) \\
& =-\frac{g h}{\sqrt{2} m_{\mathrm{K}^{*}}^{2}} \frac{m_{\mathrm{A}_{1}}}{m_{\mathrm{A}_{1}}^{2}-m_{\mathrm{K}^{*}}^{2}}\left\langle\mathrm{~B}\left|V_{\mu}^{4+i 5}\right| \mathrm{Y},\left\langle\pi^{-}\left|\partial_{\mu} \pi\right| 0\right\rangle\right. \\
& =-N \frac{g h}{\sqrt{2} m_{\mathrm{K}^{*}}^{2}} \frac{m_{\mathrm{A}_{1}}}{m_{\mathrm{A}_{1}}^{2}-m_{\mathrm{K}^{*}}^{2}} C(\mathrm{~B}, \mathrm{Y})\left(p_{\mu}^{\mathrm{Y}}-p_{\mu}^{\mathrm{B}}\right) \bar{u}_{\mathrm{B}}\left(p^{\mathrm{B}}\right) \gamma_{\mu} u_{\mathrm{Y}}\left(p^{\mathrm{X}}\right) \\
& =-i N \frac{g h}{\sqrt{\overline{2} m_{\mathrm{K}^{*}}^{2}}} \frac{m_{\mathrm{A}_{1}}}{m_{\mathrm{A}_{1}}^{2}-m_{\mathrm{K}^{*}}^{2}} C(\mathrm{~B}, \mathrm{Y})\left(m_{\mathrm{Y}}-m_{\mathrm{B}}\right) \bar{u}_{\mathrm{B}}\left(p^{\mathrm{B}}\right) u_{\mathrm{Y}}\left(p^{\mathrm{Y}}\right),
\end{aligned}
$$

according to Eqs. (24), (26), and (27), where

$$
N=\sqrt{\frac{m_{\mathbf{Y}} m_{\mathbf{B}}}{2 \omega_{\pi} E_{\mathbf{Y}} E_{\mathbf{B}}}}
$$

stands for a normalization factor, and $C(\mathrm{~B}, \mathrm{Y})$ for the $S U_{3}$ Clebsch-Gordan coefficients which are given in Table I.

TABLE I

The $S U_{3}$ Clebsch-Gordan Coefficients Defined by

$$
\left\langle\mathrm{B}\left|V_{i t}^{ \pm \pm i ;}\right| \mathrm{Y}\right\rangle=i C\left(\mathrm{~B}, \mathrm{Y}^{\prime}\right) \sqrt{\frac{m_{\mathrm{Y}} m_{\mathrm{B}}}{E_{\mathrm{Y}} E_{\mathrm{B}}}} u_{\mathrm{B}} \gamma_{\mu} u_{\mathrm{Y}}
$$

\begin{tabular}{cc}
\hline $\mathrm{Y} \rightarrow \mathrm{B} \pi$ & $C(\mathrm{~B}, \mathrm{Y})$ \\
$\Lambda \rightarrow \mathrm{p} \pi^{-}$ & $-\sqrt{\frac{3}{2}}$ \\
$\Xi^{-} \rightarrow \Lambda \pi^{-}$ & $\sqrt{\frac{3}{2}}$ \\
$\Sigma^{-} \rightarrow \mathrm{n} \pi^{-}$ & -1 \\
$\Sigma^{+} \rightarrow \mathrm{n} \pi^{+}$ & 0
\end{tabular}


The $s$-wave amplitudes $A$ for various hyperon decays then read

$$
\begin{aligned}
& A\left(\Lambda_{-}{ }^{0}\right)=\xi \sqrt{\frac{3}{2}} \frac{G_{\mathrm{F}}}{\sqrt{2}} \sin \theta \cos \theta f_{\pi} \frac{m_{\mathrm{A}_{1}}^{2}}{m_{\mathrm{A}_{1}}^{2}-m_{\mathrm{K}^{*}}^{2}}\left(m_{A}-m_{\mathrm{N}}\right), \\
& A\left(\Xi_{-}^{-}\right)=-\xi \sqrt{\frac{3}{2}} \frac{G_{\mathrm{F}}}{\sqrt{2}} \sin \theta \cos \theta f_{\pi} \frac{m_{\mathrm{A}_{1}}^{2}}{m_{\mathrm{A}_{1}}^{2}-m_{\mathrm{K}^{*}}^{2}}\left(m_{\Xi}-m_{A}\right), \\
& A\left(\Sigma_{-}^{-}\right)=\xi \frac{G_{\mathrm{F}}}{\sqrt{2}} \sin \theta \cos \theta f_{\pi} \frac{m_{\mathrm{A}_{1}}^{2}}{m_{\mathrm{A}_{1}}^{2}-m_{\mathrm{K}^{*}}^{2}}\left(m_{\Sigma}-m_{\mathrm{N}}\right), \\
& A\left(\Sigma_{\tau^{+}}\right)=0 .
\end{aligned}
$$

Using the known values for the parameters

$$
\left(f_{\pi}=134 \mathrm{MeV}, G_{\mathrm{F}}=1.026 \times 10^{-5} m_{\mathrm{p}}^{-2}\right),
$$

we compare the prediction (32) for the octet W-boson model $(\xi=\sqrt{3})$ with the experimental data in Table II. The agreement is fair. Certainly the octet W-boson model is better than any other model: For the latter the prediction is much smaller than the experimental values [12].

The result (32) is the same as that of the vector dominance model [4] as far as the relative magnitudes of various amplitudes are concerned. In fact, the first term of Eq. (24) gives the result of a simple vector dominance model. It is, therefore,

\section{TABLE II}

Comparison of Theory and Experiment ${ }^{a}$ [12] for the Nonleptonic Hyperon Decays (in the Units $10^{3} \mathrm{sec}^{-1 / 2}$ )

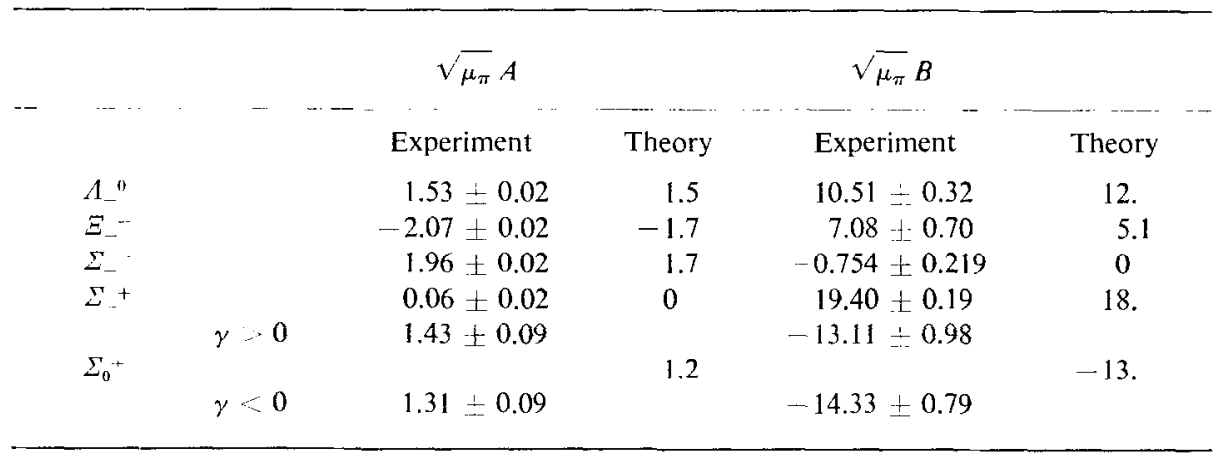

"The amplitudes $A$ and $B$ listed in the usual compilations such as in Ref. [12] correspond to $\sqrt{\mu_{\pi}} A$ and $\sqrt{\mu_{\pi}} B$ in our notation. 
essential to take into consideration the second term of Eq. (24) in order to obtain the enhancement factor $m_{\mathrm{A}_{1}}^{2} /\left(m_{\mathrm{A}_{1}}^{2}-m_{\mathrm{K}^{*}}^{2}\right)$. As was noticed in earlier works, the Lee-Sugawara relation [13]

$$
\frac{1}{\sqrt{3}} A\left(\Lambda_{-}{ }^{0}\right)+\frac{2}{\sqrt{3}} A\left(\Xi_{-}^{-}\right)+\frac{1}{\sqrt{2}}\left(A\left(\Sigma_{-}^{-}\right)-A\left(\Sigma_{+}{ }^{+}\right)\right)=0
$$

is satisfied by the amplitudes of Eq. (32) provided the Gell-Mann-Okubo mass relation is valid.

\section{THE $\mathrm{K}_{1}{ }^{0} \rightarrow 2 \pi$ DeCAY}

A similar consideration immediately leads to the $\overline{\mathrm{K}}^{0} \rightarrow \pi^{+} \pi^{-}$amplitude,

$$
\begin{aligned}
\left\langle\pi^{+} \pi^{-}\left|H_{\mathrm{int}}^{\mathrm{pv}}\right| \bar{K}^{0}\right\rangle & =h \frac{m_{\mathrm{A}_{1}}^{2}}{m_{\mathrm{A}_{1}}^{2}-m_{\mathrm{K}^{*}}^{2}}\left\langle\pi^{+}\left|v_{\mu}^{4+i 5}\right| \bar{K}^{0}\right\rangle \frac{1}{m_{\mathrm{A}_{1}}}\left\langle\pi^{-}\left|\hat{o}_{\mu} \pi\right| 0\right\rangle \\
& =i N^{\prime} \frac{g h}{\sqrt{2}} \frac{m_{\mathrm{A}_{1}}}{m_{\mathrm{A}_{1}}^{2}-m_{\mathrm{K}}^{2}} \frac{\left(\mu_{\mathrm{K}}^{2}-\mu_{\pi}^{2}\right)}{m_{\mathrm{K}^{*}}^{2}}
\end{aligned}
$$

where

$$
N^{\prime}=\frac{1}{\sqrt{8 \omega_{\pi^{+}} \omega_{\pi^{-}}-\omega_{\mathrm{K}}}}
$$

Neglecting the small $C P$ violating effect, for the $C P$ even state,

$$
\mathrm{K}_{1}{ }^{0}=\frac{i\left(\mathrm{~K}^{0}-\overline{\mathrm{K}}^{0}\right)}{\sqrt{2}},
$$

we obtain

$$
\left\langle\pi^{+} \pi^{-}\left|H_{\mathrm{int}}^{\mathrm{pv}}\right| \mathrm{K}_{1}^{0}\right\rangle=N^{\prime} g h \frac{m_{\mathrm{A}_{1}}}{m_{\mathrm{A}_{1}}^{2}-m_{\mathrm{K}^{*}}^{2}} \frac{\mu_{\mathrm{K}^{2}}-\mu_{\pi}^{2}}{m_{\mathrm{K}^{*}}^{2}} .
$$

This is not the whole story for the $\mathrm{K}_{\mathbf{1}} \mathbf{0}^{\mathbf{2}} \rightarrow 2 \pi$ decay, however. In the chiral symmetric gauge field model, we have extra terms other than Eq. (7) which contribute to the $\mathrm{K}_{\mathbf{1}}{ }^{0} \rightarrow 2 \pi$ process. In order to see this we notice [8] that the last term of Eq. (7) is the first term of the expansion in $f_{0} \Phi$ of

$$
\frac{1}{8 f_{0}^{2}} \operatorname{Tr}\left\{\left(D_{\mu} M\right)^{\dagger}\left(D_{\mu} M\right)_{\}},\right.
$$


where

$$
\begin{aligned}
M & =\frac{1+i f_{0} \Phi}{1-i f_{0} \Phi}, \\
D_{u} M & =\partial_{\mu} M-i g v_{\mu}{ }^{\mathrm{L}} M+i g M v_{\mu}{ }^{\mathrm{R}},
\end{aligned}
$$

and

$$
v_{\mu}^{\mathrm{L} . \mathrm{R}}=\frac{1}{\sqrt{2}}\left(v_{\mu} \pm a_{\mu}\right)
$$

with

$$
v_{i t}=\frac{1}{\sqrt{2}} \sum_{\alpha=1}^{8} \lambda_{\alpha} v_{\mu}{ }^{\alpha}, \quad a_{\mu}=\frac{1}{\sqrt{2}} \sum_{\alpha=1}^{8} \lambda_{\alpha} a_{\mu}{ }^{a} .
$$

That is, in the expansion

$$
\begin{aligned}
& \frac{1}{8 f_{1)^{2}}{ }^{2}} \operatorname{Tr}\left\{\left(D_{\mu} M\right)^{\dagger} D_{\mu} M\right\} \\
& =\frac{1}{2} \operatorname{Tr}\left(\partial_{\mu} \Phi-\frac{g}{\sqrt{2} f_{0}} a_{\mu}\right)^{2}-\frac{i g}{\sqrt{2}} \operatorname{Tr}\left\{\left(\partial_{\mu} \Phi-\frac{g}{\sqrt{2} f_{0}} a_{\mu}\right)\left[l_{\mu}, \Phi\right]\right\}+\cdots,
\end{aligned}
$$

the second term contains terms responsible for the decay $\mathrm{K}_{1}{ }^{0} \rightarrow \pi^{+} \pi^{-}$,

$$
\begin{aligned}
-\frac{i g}{\sqrt{2}} \operatorname{Tr}\left\{\left(\partial_{n} \Phi-\frac{g}{\sqrt{2} f_{0}} a_{\mu}\right)\left[v_{\mu}, \Phi\right]\right\} \\
=-\frac{g}{4}\left(\partial_{\mu} \varphi-\frac{g}{\sqrt{2} f_{0}} a_{\mu}\right)^{7}\left(v_{\mu}^{4+i 5} \varphi^{1-i 2}+\text { h.c. }\right) \\
+\frac{g}{4} \phi^{7}\left\{v_{\mu}^{4+i 5}\left(\partial_{\mu} \varphi-\frac{g}{\sqrt{2} f_{0}} a_{\mu}\right)^{1-i 2}+\text { h.c. }\right\} .
\end{aligned}
$$

Note that since

$$
\phi^{\mathbf{K}^{0}}=\frac{1}{\sqrt{2}} \varphi^{\mathbf{6 - i 7}}
$$

$\varphi^{\mathrm{K}_{1}{ }^{0}}$ transforms as $\varphi^{7}$ and $\varphi^{\mathrm{K}_{2}{ }^{0}}$ as $\varphi^{6}$, where

$$
\mathrm{K}_{2}{ }^{0}=\frac{1}{\sqrt{2}}\left(\mathrm{~K}^{0}+\overline{\mathrm{K}}^{0}\right)
$$

is the $C P$ odd state.

The weak interaction Lagrangian

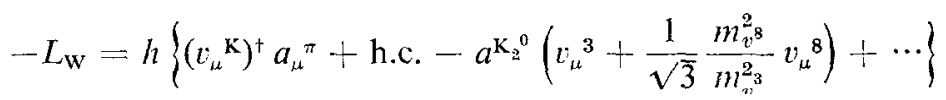


mixes the $a_{\mu}^{\mathrm{K}_{2}{ }^{0}}$ field with $\rho, \omega$, or $\phi$, but not the $a_{\mu}^{\mathrm{K}_{1}{ }^{0}}$ field. Therefore, it is sufficient to consider the strong mixing of the $\mathrm{K}_{1}{ }^{0}$ field and $a_{\mu}^{\mathrm{K}_{1}{ }^{0}}$ field in the gauge field Lagrangian,

$$
-L_{\mathrm{G}^{\mathrm{K}}}^{\mathrm{K}^{0}}=\frac{1}{2} m_{\mathrm{K}^{*}}^{2}\left(a_{\mu}^{\mathrm{K}_{1}{ }^{0}}\right)^{2}+\frac{1}{2}\left(\partial_{\mu} \varphi^{\mathrm{K}_{1}{ }^{0}}-\frac{g}{\sqrt{2} f_{0}} a_{\mu}^{\mathrm{K}_{1}{ }^{0}}\right)^{2}+\frac{1}{2} \bar{\mu}_{\mathrm{K}}{ }^{2}\left(q^{\mathrm{K}_{1}{ }^{0}}\right)^{2}
$$

$\bar{\mu}_{\mathrm{K}}$ being the bare mass of $\mathrm{K}^{0}$ particle. Equation (46) can be diagonalized by

$$
a_{\mu}^{\mathrm{K}_{1}{ }^{0}}=A_{\mu}^{\mathrm{K}_{1}{ }^{0}}+\frac{m_{\rho}}{m_{\mathrm{K}^{*}} m_{\mathrm{K}_{\mathrm{A}}}} \partial_{\mu} \mathrm{K}_{\mathbf{1}}{ }^{0}
$$

and

$$
\varphi^{7} \equiv \varphi^{K_{1}^{0}}=\sqrt{Z_{\mathbf{K}}} K_{1}^{0},
$$

where

$$
m_{\mathbf{K}_{\mathrm{A}}}^{2}=m_{\mathrm{K}^{*}}^{2}+m_{\rho}^{2}
$$

and

$$
\sqrt{Z_{\mathrm{K}}}=\frac{m_{\mathrm{K}_{\mathrm{A}}}}{m_{\mathrm{K}^{*}}} .
$$

The physical mass of $\mathrm{K}^{0}$ is then

$$
\mu_{\mathrm{K}}=\sqrt{\bar{L}_{\mathrm{K}}} \bar{\mu}_{\mathrm{K}}=\frac{m_{\mathrm{K}_{\mathrm{A}}}}{m_{\mathrm{K}^{*}}} \bar{\mu}_{\mathrm{K}} .
$$

According to Eqs. (15), (16), and (44)-(49), we have an additional interaction Hamiltonian for the process $\mathrm{K}_{1}{ }^{0} \rightarrow \pi^{+} \pi^{-}$,

$$
\begin{aligned}
H_{\text {trilinear }}= & \frac{g}{\sqrt{2}} \frac{h}{m_{\mathrm{K}^{*}}^{2} m_{\mathrm{A}_{\mathbf{x}}}}\left\{\frac{m_{\mathrm{K}^{*}}}{m_{\mathrm{K}_{\mathrm{A}}}} \partial_{\mu} \mathrm{K}_{1}{ }^{0}\left(\partial_{\mu} \pi^{\prime} \pi+\pi^{\dagger} \partial_{\mu} \pi\right)\right. \\
& -\frac{m_{\mathrm{K}_{\mathrm{A}}}}{m_{\mathrm{K}^{*}}} \mathrm{~K}_{1}{ }^{*} \partial_{\mu} \pi^{\dagger} \partial_{\mu} \pi^{\prime},
\end{aligned}
$$

which gives the matrix element

$$
\begin{aligned}
\left\langle\pi^{+} \pi^{-}\left|H_{\text {trillnear }}\right| \mathrm{K}_{1}{ }^{0}\right\rangle & =N^{\prime} \frac{g h}{\sqrt{2} m_{\mathrm{K}^{*}} m_{\mathrm{A}_{1}} m_{\mathrm{K}_{\mathrm{A}}}}\left\{\left(p_{\mu}{ }^{\mathrm{K}}\right)^{2}+\left(\frac{m_{\mathrm{K}_{\mathrm{A}}}}{m_{\mathrm{K}^{*}}}\right)^{2}\left(p_{\mu}^{\pi^{+}} p_{\mu}^{\pi^{-}}\right)\right\} \\
& =N^{\prime} \frac{g h \mu_{\mathrm{K}^{2}}{ }^{2}}{\sqrt{2} m_{\mathrm{K}^{*}} m_{\mathrm{A}_{1}} m_{\mathrm{K}_{\mathrm{A}}}}\left\{1+\frac{1}{2}\left(\frac{m_{\mathrm{K}_{\mathrm{A}}}}{m_{\mathrm{K}^{*}}}\right)^{2}\left(1-\frac{2 \mu_{\pi^{2}}}{\mu_{\mathrm{K}}{ }^{2}}\right)\right\} .
\end{aligned}
$$


Combining Eqs. (37) and (52), we conclude that

$$
\left\langle\pi^{+} \pi^{-}\left|H_{\text {int }}^{\text {pv }}+H_{\text {trilinear }}\right| \mathrm{K}_{1}{ }^{\mathrm{U}}\right\rangle=N^{\prime} A\left(\mathrm{~K}_{\mathbf{1}}{ }^{0} \rightarrow \pi^{+} \pi^{-}\right),
$$

where

$$
\begin{aligned}
& A\left(\mathrm{~K}_{1}{ }^{0} \rightarrow \pi^{+} \pi^{-}\right) \\
& =\frac{g h \mu_{\mathrm{K}}^{2}}{m_{\mathrm{K}^{*}}^{2} m_{\Lambda_{1}}}\left\{\frac{m_{\mathrm{A}_{1}}^{2}}{m_{\mathrm{A}_{1}}^{2}-m_{\mathrm{K}^{*}}^{2}}-\left(1-\frac{\mu_{\pi}^{2}}{\mu_{\mathrm{K}^{2}}^{2}}\right)\right. \\
& \left.-\frac{1}{\sqrt{2}} \frac{m_{\mathrm{K}^{*}}}{m_{\mathrm{K}_{\mathrm{A}}}}\left[1+\frac{1}{2}\left(\frac{m_{\mathrm{K}_{\mathrm{A}}}}{m_{\mathrm{K}^{*}}}\right)^{2}\left(1-\frac{2 \mu_{\pi}^{2}}{\mu_{\mathrm{K}^{2}}{ }^{2}}\right)\right]\right\} \\
& =\xi G_{\mathrm{F}} \sin \theta \cos \theta f_{\pi} \mu_{\mathrm{K}^{2}}{ }^{2}\left\{\frac{m_{\mathrm{A}_{1}}^{2}}{m_{\Lambda_{1}}^{2}-m_{\mathrm{K}^{*}}^{2}}-\frac{1}{\sqrt{2}} \frac{m_{\mathrm{K}^{*}}}{m_{\mathrm{K}_{\mathrm{A}}}}\left(1+\frac{1}{2}\left(\frac{m_{\mathrm{K}_{\mathrm{A}}}}{m_{\mathrm{K}^{*}}}\right)^{2}\right)\right\} \text {. }
\end{aligned}
$$

In the last expression, the pion mass has been neglected.

The numerical result is given in Table III. The second term of Eq. (54), which

TABLE III

$$
\begin{aligned}
& \text { Comparison of Theory and Experiment for } \\
& \text { the } \mathrm{K}_{1}{ }^{0} \rightarrow 2 \pi \text { Decay (in the Units } 10^{-4} \text { ) }
\end{aligned}
$$

\begin{tabular}{ccc}
\hline & Experiment & Theory \\
\hline$A\left(\mathrm{~K}_{1}{ }^{0} \rightarrow \pi^{+} \pi^{-}\right)$ & $3.98 \pm 0.02$ & 3.5 \\
$A\left(\mathrm{~K}_{1}{ }^{10} \rightarrow \pi^{\prime \prime} \pi^{0}\right)$ & $2.69 \pm 0.03$ & 2.5 \\
\hline
\end{tabular}

stems from the additional Hamiltonian $H_{\text {trilinear }}$, is responsible for bringing the theoretical prediction closer to the experimental data. The $\Delta I=\frac{1}{2}$ rule, of course, predicts

$$
A\left(\mathrm{~K}_{1}{ }^{0} \rightarrow \pi^{0} \pi^{0}\right)=\frac{1}{\sqrt{2}} A\left(\mathrm{~K}_{1}{ }^{0} \rightarrow \pi^{+} \pi^{-}\right) .
$$

\section{The $P$-Wave Amplitudes}

The sum rule between the $s$-wave and $p$-wave amplitudes of the nonleptonic hyperon decay has been derived by using PCAC, current algebra, and the infinite momentum technique [14]. The agreement of the theory and experiment is excellent 
if the $s$-wave amplitudes are taken as an input information. In this scction, nevertheless, we use the sum rule of Ref. [14] and the theoretical result for the $s$-wave amplitudes obtained in this article, Eq. (32), in order to make a complete set of theoretical predictions.

Since the $s$-wave amplitudes, Eq. (32), satisfies the Lee-Sugawara relation, Eq. (33), and the constraint $A\left(\Sigma_{T^{+}}{ }^{+}\right)=0$, the sum rule [14] reads

$B\left(\Sigma_{+}^{*}\right)=g_{A} \frac{m_{\Sigma}+m_{\mathrm{N}}}{m_{\Sigma}-m_{\mathrm{N}}} A\left(\Sigma_{-}\right)=\xi g_{A} \frac{G_{\mathrm{F}}}{\sqrt{2}} \sin \theta \cos \theta f_{\pi}^{\prime} \frac{m_{\mathrm{A}_{1}}^{2}}{m_{\mathrm{A}_{1}}^{2}-m_{\mathrm{K}^{*}}^{2}}\left(m_{\Sigma}+m_{\mathrm{N}}\right)$, $B\left(\Sigma_{-}^{-}\right)=0$

$$
\begin{aligned}
& B\left(\Lambda_{\mathbf{0}^{-}}\right)=g_{A} \frac{m_{\Lambda}+m_{\mathrm{N}}}{m_{A}-m_{\mathrm{N}}} A\left(A_{--}^{0}\right)-\sqrt{\frac{2}{3} D \frac{m_{\Sigma}+m_{\mathrm{N}}}{m_{\Sigma}-m_{\mathrm{N}}}} A\left(\Sigma_{-}\right) \\
& =\xi g_{A} \sqrt{\frac{3}{2}} \frac{G_{\mathrm{F}}}{\sqrt{2}} \sin \theta \cos \theta f_{\pi} \frac{m_{\mathrm{A}_{\mathrm{I}}}^{2}}{m_{\mathrm{A}_{1}}^{2}-m_{\mathrm{K}^{*}}^{2}}\left\{m_{A}+m_{\mathrm{N}}-\frac{2}{3} \frac{D}{D+F}\left(m_{\Sigma}+m_{\mathrm{N}}\right)\right\},
\end{aligned}
$$

and

$$
\begin{aligned}
& B\left(\Xi_{-}^{-}\right)=-(D-F) \frac{m_{\Xi}+m_{\Lambda}}{m_{\Xi}-m_{\Lambda}} A\left(\Xi_{-}^{-}\right) \\
& =\xi(D-F) \sqrt{\frac{3}{2}} \frac{G_{\mathrm{F}}}{\sqrt{2}} \sin \theta \cos \theta f_{\pi} \frac{m_{\mathrm{A}_{1}}^{2}}{m_{\mathrm{A}_{\mathrm{\lambda}}}^{2}-m_{\mathrm{K}^{*}}^{2}}\left(m_{\xi}+m_{\Lambda}\right),
\end{aligned}
$$

where the parameters are given by [3]

$$
D=0.74 \pm 0.02, \quad F=0.49 \pm 0.02,
$$

and

$$
g_{\mathrm{A}}=D+F=1.23 \pm 0.03
$$

We notice that the amplitude $B\left(\Lambda_{-}{ }^{0}\right)$ is expressed as

$$
B\left(\Lambda_{-}^{0}\right)=\left\{g_{A} A\left(\Lambda_{-}^{0}\right)-\sqrt{\frac{2}{3}} D A\left(\Sigma_{-}^{-}\right)\right\} \frac{m_{\Lambda}+m_{\mathrm{N}}}{m_{\Lambda}-m_{\mathrm{N}}}
$$

in Ref. [14]. This formula, however, was obtained in the soft pion limit, and the limit $m_{\Sigma}-m_{\Lambda}=0$, so that we encounter the ambiguity concerning the masses of $\Sigma$ and $A$ in using the sum rule. We have chosen Eq. (55) for the amplitude $B\left(\Lambda_{-}^{0}\right)$ since it leads to a simpler result.

The numerical estimate of Eq. (55) is listed in the last column of Table II. We emphasize that the theoretical prediction of Table II has no adjustable parameter. 
Finally we give a comment on the Lee-Sugawara relation for the $p$-wave amplitudes. The validity of such a relation implies the relation

$$
\frac{D}{F}=\frac{3}{2} \frac{\left(2 m_{\Sigma}+m_{\Lambda}+m_{\Sigma}\right)}{\left(4 m_{\Sigma}+3 m_{1}-3 m_{\Sigma}\right)}=1.47,
$$

according to Eq. (55). The left side gives $D / F=1.51 \pm 0.10$, which is remarkably close to the value of the right side. In the $S U_{3}$ symmetric limit, Eq. (57) reduces to

$$
\left(\frac{D}{F}\right)_{S U_{3} \text { symmetriclimit }}=\frac{3}{2} .
$$

This is the same value as in the $S U_{6}$ theory [15].

In conclusion, our analysis favors the weak interaction Lagrangian based on the octet $\mathrm{W}$ bosons. ${ }^{2}$

\section{ACKNOWLEDGMENT}

It is a pleasure to thank Professor K. Nishijima who gave the author stimulation on the subject. Thanks are also due to Professors O. E. Overseth, Marc H. Ross, Y. P. Yao, and Dr. I. Kimel for useful discussions, and Professor D. N. Williams for reading the manuscript.

\section{REFERENCES}

1. Y. Tomozawa, Phys. Lett. B 30 (1969), 543; Phys. Rev. 2 D (1970), 1586.

2. M. Gell-Mann and M. Levy, Nuovo Cimento 16 (1960), 705; N. Cabibbo, Phy's. Rev. Lett. 10 (1963), 531.

3. H. Fil.thuth, in "Topical Conference on Weak Interactions" (J. S. Bell, Ed.), p. 131, CERN, 1969.

4. J. J. Sakurai, Phys. Rev. 156 (1967), 1508.

5. K. Nishisima and H. Sato, in "Proc. of Coral Gables Conference on Fundamental Interactions at High Energy" (A. Perlmutter et al., Eds.) p. 293, Gordon and Beach, 1970; Progr. Theor. Phys. 43 (1970), 1316.

6. C. N. Yang and R. L. Mills, Phys. Rev. 96 (1954), 191; R. Utiyama, Phys. Rev. 101 (1956), 1597; T. D. Lee and B. Zumino, Phy's. Rev. 163 (1967), 1667; J. Wess and B. Zumino, Phys. Rev. 1.63 (1967), 1727.

7. S. Gasiorowicz and D. A. Geffen, Rev. Mod. Phys. 41 (1969), 531. This review article contains some of the earlier references.

8. K. Kawarabayashi, "Lectures in Theoretical Physics," Vol. XI-A; "Elementary Particle Physics," Part 1. (K. Mahantappa et al., Eds.), p. 227, University of Colorado, 1968.

${ }^{2}$ The same conclusion has been reached by I. Kimel [16], who used the method of the spectral function sum rule. 
9. J. J. Sakural, Ann. Phys. (New York) 11 (1960), 1; M. Gell-Mann and F. Zachariasen, Phys. Rev. 124 (1961), 953; M. Gell-Mann, D. Sharp, and W. G. Wagner, Phys. Rev. Lett. 8 (1962), 261; Y. Nambu and J. J. Sakurai, Phys. Rev. Lett. 8 (1962), 79; N. M. Kroll, T. D. LeE, ANd B. Zumino, Phys. Rev. 157 (1967), 1376; 163 (1967), 1667.

10. S. Weingerg, Phys. Rev. Lett. 18 (1967), 507.

11. K. Kawarahayashi and M. Suzuki, Phys. Rev. Lett. 16 (1966), 225; Riazuddin and FayYazUdDin, Phys. Rev. 147 (1966), 1071.

12. The experimental data is a compilation due to O. E. Oversstil, private communication. See also H. Filthuth, in "Topical Conference on Weak Interactions" (J. S. Bell, Eds.), p. 131, CERN, 1969; J. P. Berge, "Proc. of the XIII-th International Conference on High Energy Physics" (E. M. McMillan, Ed.), p. 46, 1966.

13. H. Sugawara, Progr. Theor. Phys. 31 (1964), 213; B. W. Lee, Phy's. Rev. Lett. 12 (1964), 83; M. Gell-Mann, Phys. Rev. Lett. 12 (1964), 155; S. Oкubo, Phys. Lett. 8 (1964), 362.

14. Y. Tomozawa, Phys. Lett. 32 B (1970), 485.

15. F. Gürsey and L. A. Radicati, Phys. Rev. Lett. 13 (1964), 173; B. Sakita, Phy's. Rev. B 136 (1964), 1756; F. Gürsey, A. Pais, And L. A. Radicati, Phy's. Rev. Lett. 13 (1964), 299. 16. I. Kimel, to be published. 